This item is the archived peer-reviewed author-version of:

Comparison of seed morphology of two ginkgo cultivars

\title{
Reference:
}

Tian Fang, Wang Yujun, Sandhu Hardev S., Gielis Johan, Shi Peijian.- Comparison of seed morphology of tw o ginkgo cultivars Journal of Forestry Research - ISSN 1007-662X - (2018), p. 1-8

Full text (Publisher's DOI): https://doi.org/10.1007/S11676-018-0770-Y

To cite this reference: https://hdl.handle.net/10067/1549870151162165141 


\section{Comparison of seed morphology of two ginkgo cultivars}

Fang Tian ${ }^{1 a} \cdot$ Yujun Wang $^{1 a} \cdot$ Hardev S. Sandhu ${ }^{b} \cdot$ Johan Gielis ${ }^{c} \cdot$ Peijian Shi $^{2 a}$

a Co-Innovation Centre for Sustainable Forestry in Southern China, Nanjing

Forest University, Nanjing 210037, Jiangsu, People's Republic of China

b Everglades Research and Education Center, University of Florida, 3200 E.

Palm Beach Road, Belle Glade, FL 33430, USA

c Department of Biosciences Engineering, University of Antwerp, Antwerp

B-2020, Belgium

${ }^{1}$ These two authors contributed equally to this work.

${ }^{2}$ Corresponding author. Email address: peijianshi@gmail.com. Tel: + 862585427

231. 
Abstract Ginkgo biloba L. is a precious relic tree species with important economic value. Seeds, as a vital reproductive organ of plants, can be used to distinguish cultivars of the species. We chose 400 seeds from two cultivars of ginkgo ("Fozhi" and "Maling"; 200 seeds for each cultivar) as the study material and used the Gielis equation (Gielis 2003; 2017) to fit the projected shape of these seeds. The coefficients of variation (CV) in root mean squared errors (RMSE) obtained from the fitted data were used to compare the level of inter-cultivar variations in seed shape. We also used the covariance analysis to compare the allometric relationships between seed weights and projected areas of these two cultivars. The Gielis equation fitted well the seed shapes of two ginkgo cultivars. The lower CV in RMSE of cultivar "Fozhi" than "Maling" indicated a less symmetrical seed shape in the latter than the former. The bootstrap percentile method (Sandhu et al., 2011) showed that the seed shape differences between the two cultivars were significant. However, there was no significant difference in the exponents between the seed weights and the projected areas of these two cultivars. Overall, the significant differences in shapes between the seeds of two ginkgo cultivars were well explained by the Gielis equation; this model can be further extended to compare morphological differences in other ginkgo cultivars, and even for plant seeds or animal eggs that have similar oval shapes.

Keywords allometry $\cdot$ curve fitting $\cdot$ coefficient of variation $\cdot$ Gielis equation root mean squared errors 


\section{Introduction}

Ginkgo biloba L., a relic tree species that is native to China, has a living history of more than 300 million years and is known as a "living fossil" (Mahadevan et al., 2008; Zhou et al., 2003). It belongs to the family Ginkgoaceae that now has only one existing species left, which has been widely planted for timber, medical, and ornamental utilizations (Nakanishi, 2005; Tredici, 1991). (Fig. S1 in online supplementary material exhibits the ginkgo trees along one side of a road.)

China is one of the world's largest producers of ginkgo seeds and its production rate has increased year after year. G. biloba fruits are extensively studied because the kernels contain a great deal of nutritional and bioactive compositions that are of vital dietary and medicinal value (Hu et al., 2014). Moreover, the Pharmacopoeia of the People's Republic of China (2015 Edition) stated that ginkgo leaves can promote blood circulation and reduce blood fat (Zhang et al., 2016). Its extracts are added into tea or a functional beverage to improve human health and is also made into new medical products such as GinkgoPlus, Topnin, Promod, Tanakan or cosmetic products (Cheng and Zhang, 2008).

In China, ginkgos are classified into the following cultivars: Changzi, Fozhi, Maling, Meihe, Yuanzi (or called Longyan) (He, 1989). Among these cultivars, Fozhi (meaning the Buddha's finger in Chinese) and Maling (meaning horse's bell in Chinese) seeds are widely planted and the dominant cultivars in Jiangsu

Province. However, accurate morphological differences among these cultivars have 
long been overlooked. Fozhi is named because their seeds have a long-ovate or fingerlike spindle shape; whereas Maling seeds appear to have a broader shape. In taste, Fozhi seeds are juicy and sweet, while Maling seeds are waxy and sweet (He, 1989). Besides shape and taste, one ovule of Maling can usually develop into fruit, while two ovules of Fozhi can develop into two fruits.

Traditionally, ginkgo seeds are visually classified according to their size and morphology of kernels. Moreover, to our knowledge, morphological characteristics are all determined by manual measurements that carry errors due to their irregular shapes, and there is no way to make an accurate description of seed shape for its morphogenesis and classification. Many researchers have uncovered the underlying physical and morphological properties of natural plants by using different mathematical models.

Mirzaee et al. (2008) found that the best and worst models for predicting the mass of two varieties of apricot based on some physical characteristics. Jahromi et al. (2008) determined dimensions and projected areas by surveying some physical properties of dates (cv. Dairi) with image processing techniques. Models can be built to distinguish the differences between native (Celastrus scandens L.) and invasive ( $C$. orbiculatus Thunb.) bittersweet species by determining the morphological characteristics of leaf and fruit (Leicht-Young et al., 2007).

To elucidate biophysical processes of underlying plant morphology, some investigators (Kincaid and Schneider, 1983; Kuhl and Giardina, 1982) analyzed many complex forms using the elliptic Fourier analysis method, which has been 
applied to study plant leaves (McLellan, 1993). Starting in the 1990s, geometric morphometrics developed rapidly in biological research (Bookstein, 1996; Gielis, 2003, 2017; Rohlf, 1996; Jensen, 1990). For example, mathematical models can be used to describe the actual patterning in shells (Meinhardt 1998) and whorl morphogenesis in dasycladalean algae (Dumais and Harrison, 2000). However, it has a disadvantage in that an algorithm cannot exactly describe a real plant (Van Oystaeyen et al., 1996).

Gielis $(2003 ; 2017)$ has put forward a universal bio-geometric formula that can describe various geometric shapes and forms of creatures and their organs. Many researchers take advantage of the general Gielis equation or its transformation according to their actual needs. The original formula is used to describe the cross-sectional shapes of the leaf petiole (Faisal et al., 2010). Simplified versions of the Gielis equation can depict the special shapes more effectively, especially for the shapes of tree-rings and bamboo leaves (Chacón, 2006; Lin et al., 2016; Shi et al., 2015b), which contribute to the understanding of underlying laws for plant biological phenomena.

In the present study, we attempted to use the Gielis equation to simulate the seed shapes of ginkgo cultivars, which can be used to identify the morphological differences between the two cultivars. A new method using the coefficient of variation is also proposed to check the extent of intra-cultivar seed shape variation. 


\section{Materials and methods}

\section{Materials}

Two cultivars of G. biloba (Fozhi and Maling) were selected from Taixing of Jiangsu Province $\left(32^{\circ} 12^{\prime} 58.46^{\prime \prime} \mathrm{N}, 120^{\circ} 01^{\prime} 12.69^{\prime \prime} \mathrm{E}\right)$ to conduct this study. Ten trees for every cultivar were randomly chosen from their plantation, and 20 healthy seeds per tree were collected when they attained commercial maturity (fruit starting to drop off trees naturally) in late October of 2016.

\section{Basic data collection}

We measured fresh seed weights using an electronic scale with $0.1 \mathrm{mg}$ precision (ME204/02; Mettler Toledo instruments Co., Ltd., Shanghai, China) and their thickness by using a vernier caliper (CD-20APX; Mitutoyo Corporation, Kanagawa, Japan). The projection shapes of seeds were scanned by a photo scanner (HP Scanjet 4850; Hewlett-Packard Company, Palo Alto, California, USA) and their images were converted into bmp (Bitmap) format. For further analysis, we used the MATLAB program proposed by Shi et al. (2015a) to extract the edge data of the projection shapes of seeds in Cartesian coordinates.

\section{Morphological model}

Gielis $(2003,2017)$ proposed a general equation that can be used to describe the shapes of creatures including their organs: 
$r(\varphi)=\left(\left|\frac{1}{A} \cos \left(\frac{m}{4} \varphi\right)^{n_{2}}+\right| \frac{1}{B} \sin \left(\frac{m}{4} \varphi\right)^{n_{3}}\right)^{-\frac{1}{n_{1}}}$

Eq. (1)

Here, $r$ and $\varphi$ are polar coordinates of the edge of an object on the plane; $A, B, n_{1}$, $n_{2}$ and $n_{3}$ are positive constants; $m$ is a positive integer, which determines the number of protruding angles in an edge's shape. For the ginkgo seed, there are two obvious angles, so $m=2$. However, there are still too many parameters that can affect the efficiency of curve fitting and the accuracy of parameters estimated. To solve these problems, we re-parameterized the original Gielis equation as (see Appendix S2 in the online supplementary material for details):

$r(\varphi)=a\left(\left|\cos \frac{\varphi}{2}\right|^{n_{2}}+\frac{1}{k}\left|\sin \frac{\varphi}{2}\right|^{n_{3}}\right)^{-\frac{1}{n_{1}}}$

Eq. (2)

where $a=A^{n_{2} / n_{1}}$ and $k=B^{n_{3}} / A^{n_{2}}$. For simplicity, we refer to Eq. [2] as the Gielis equation in this manuscript.

Based on the re-parameterized Gielis equation, we can easily calculate the planar projection area $(S)$, and length $(L)$ of ginkgo seeds that is defined as the distance from the left edge to the right edge on the major axis.

$$
S=\frac{1}{2} \int_{0}^{2 \pi}[r(\varphi)]^{2} d \varphi
$$

Eq. (3)

$$
L=\varphi(0)+\varphi(\pi)=a+a \cdot \sqrt[n_{1}]{k}
$$

Eq. (4)

The width can be estimated using the numerical method. After simulating many 
points by the Gielis equation (e.g. $\varphi$ ranges from 0 to $2 \pi$ in $2 \pi / 2000$ intervals), we can use the 2-fold maximum $y$ value on the plane to approximate the width.

We used root mean squared error (RMSE) to evaluate the model's goodness of fit, and RMSE is related to the squared sum of residual sum of squares (RSS): $\mathrm{RSS}=\sum_{i=1}^{n}\left(r_{i}-\hat{r}_{i}\right)^{2}$

Eq. (5)

$\mathrm{RMSE}=\sqrt{\mathrm{RSS} / n}$

Eq. (6)

$r_{i}$ represents the observed distance from the polar point to the $i$-th point on the edge; and $\hat{r}_{i}$ represents the fitted distance from the polar point to the $i$-th point on the edge; and $n$ represents sample size of points (namely the number of sampled points determined by the scanned image resolution).

Here, RMSE represents the average measure of the deviances between the observed and fitted distances. However, RMSE cannot be directly used to compare the intra-variations of seed projection shapes because it is affected by the model's goodness of fit and the seed size. Thus, we used the coefficient of variation $(\mathrm{CV})$ in $\mathrm{RMSE}$ to reflect the intra-cultivar variation in the projected shapes, and then we also used the CV values calculated from two groups of RMSE to compare the inter-cultivar differences:

$\mathrm{CV}_{\mathrm{RMSE}}=\frac{\mathrm{SD}_{\mathrm{RMSE}}}{\mathrm{MEAN}_{\mathrm{RMSE}}}$

Eq. (7)

$\mathrm{SD}_{\mathrm{RMSE}}$ represents the standard deviation of RMSE; MEAN $\mathrm{RMSE}_{\text {represents the }}$ 
mean of RMSE.

\section{Allometric relationship between seed weight (w) and projection area (A)}

$W=\alpha A^{\beta}$

Eq. (8)

where $\alpha$ and $\beta$ are constants with $\beta$ theoretically being equal to $2 / 3$ if the object density is regular. However, this exponent might not be equal to $2 / 3$ because of the density irregularity. Eq. [8] can be rewritten as:

$\ln (W)=\ln (\alpha)+\beta \ln (A) \Leftrightarrow y=c+\beta x$

Eq. (9)

We used the bootstrap percentile method (Sandhu et al., 2011) to check whether the slope estimates between the two cultivars have significant differences.

\section{Statistical analyses}

All data analyses and figures were conducted using the statistical software $\mathrm{R}$

(version 3.1.3; R Development Core Team, 2015). An R script for implementing the curve fitting by using the Gielis equation is provided in the attached Appendix S3.

\section{Results}

The projection edges of the two ginkgo cultivar seeds exhibited similar oval shapes (Fig. 1a and c). The predicted projection shapes (Fig. 1b and d) of two 
cultivar seeds by the Gielis equation matched well with the scanned projection shapes. Both medians of RMSEs that were used to measure the goodness of fit of curve fitting for two cultivar seeds were approximately equal to 0.01 , which demonstrated the validity of the model (see Fig. 1e).

Although there was significant difference in RMSE between these two cultivars $(p<0.01)$, the differences in the goodness of fit were mainly caused by the differences of projection areas (Fig. 2c). The CV in RMSE of cultivar Fozhi was significantly lower than that of cultivar Maling (21.6\% versus $25.6 \%)$. Clearly, the $95 \%$ confidence interval (CI) of the differences in the CV bootstrap replicates between two cultivars, $(-7.0 \%,-0.7 \%)$ did not include 0 (Fig. 1f). It demonstrated that the intra-cultivar variation in 2-D profile of cultivar Fozhi is lower than that of cultivar Maling. 

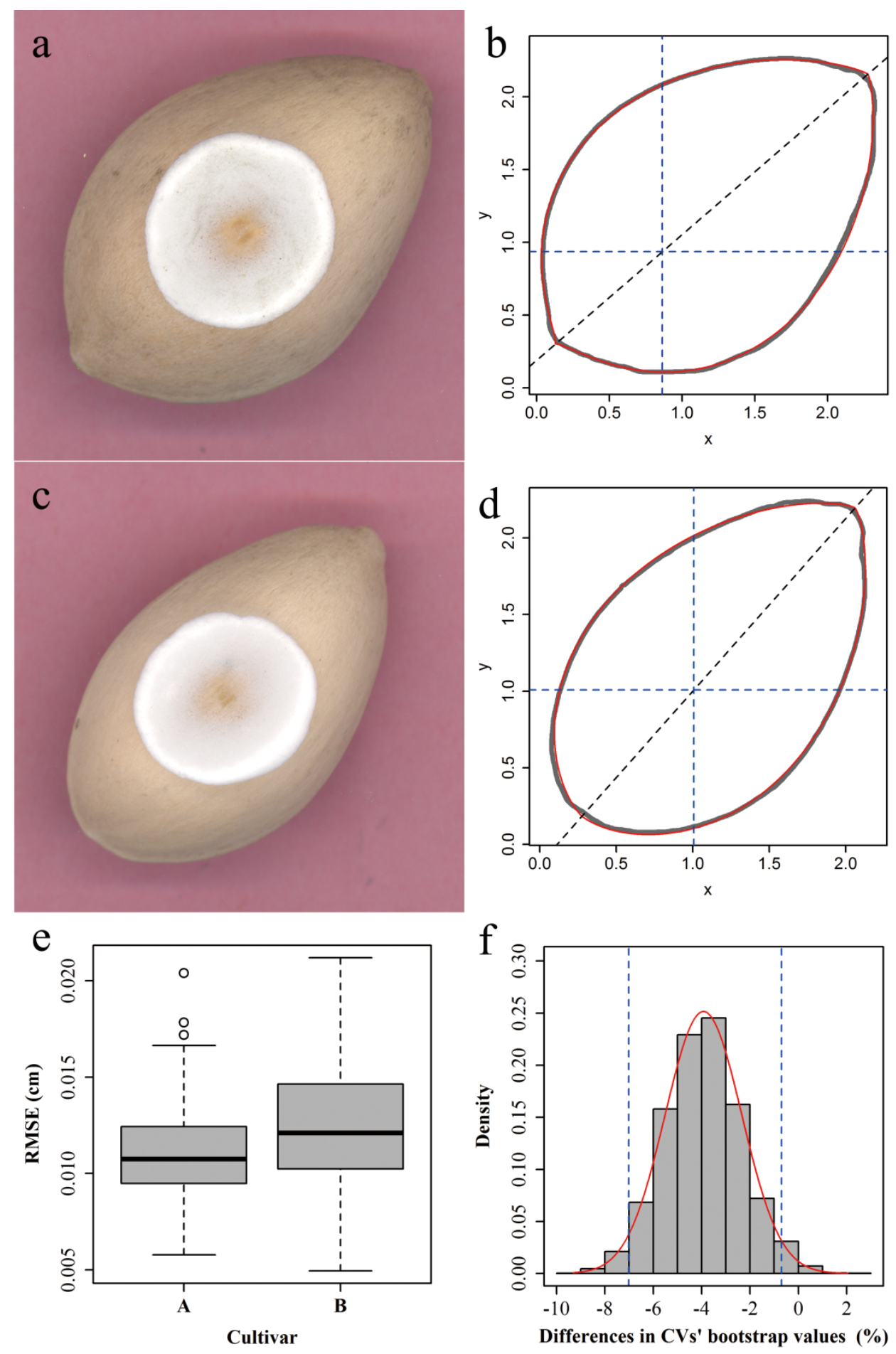

Fig. 1. Examples of projected shape fitting and model evaluation between the two cultivars. (a-b) The original shape (gray lines) and the fitting shape (red lines) of cultivar Maling. (c-d) The original shape and the fitting shape of cultivar Fozhi. (e) The root mean squared errors (RMSE) of two cultivars. (f) The density 
distribution differences in $\mathrm{CV}_{\mathrm{RMSE}}$ between the two groups of 5,000 bootstrap replicates, A represents cultivar Fozhi, and B represents cultivar Maling.

For the five representative parameters (seed length, width, thickness, projection area, and fresh weight), the differences between the two cultivars were all significant $(p<0.01)$ except for the length $(p=0.106$; Fig. $2 \mathrm{a})$. The seed width, thickness, projection area, and fresh weight of cultivar Fozhi were smaller than those of cultivar Maling (Fig. 2b-e). 

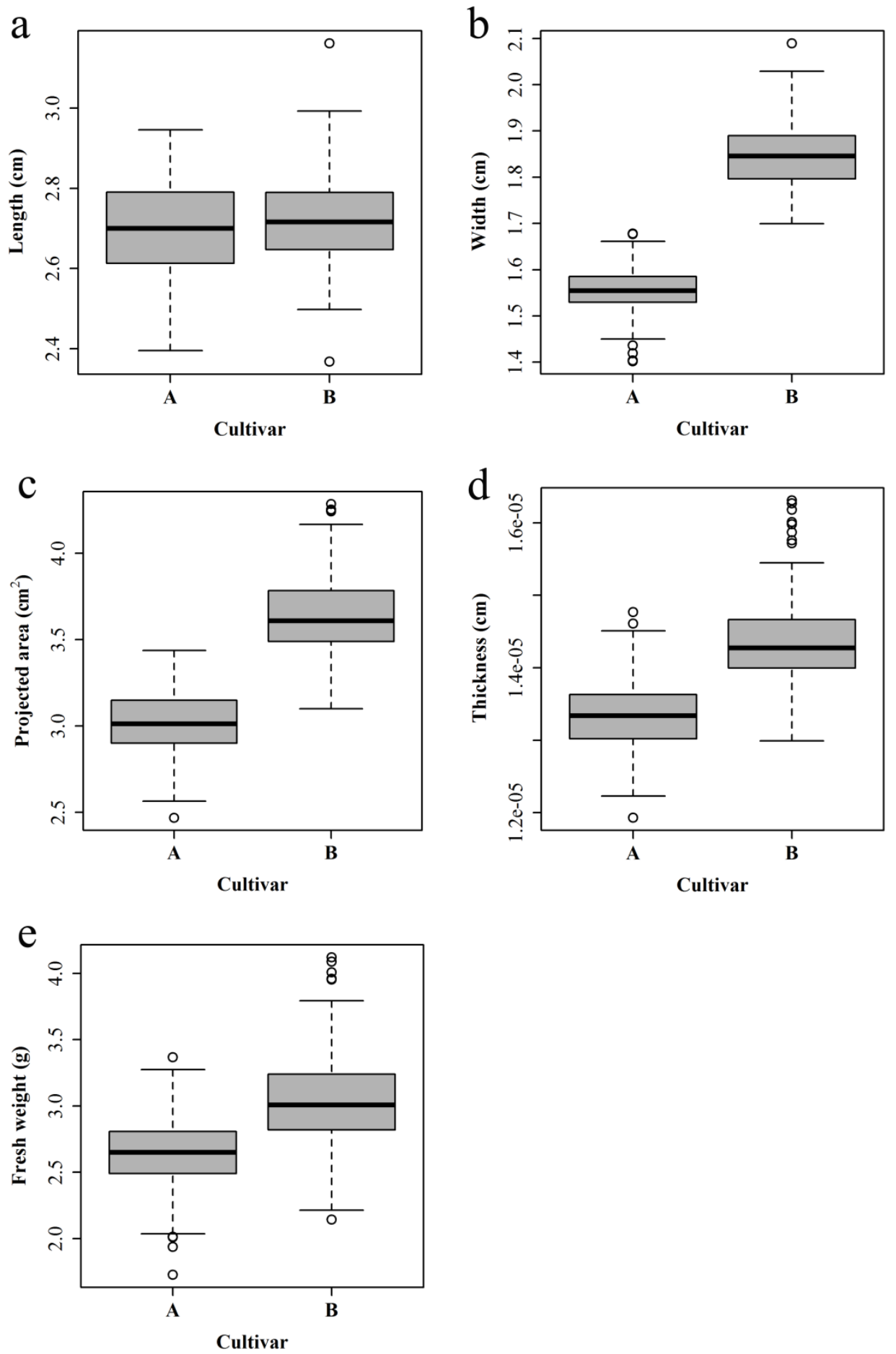

Fig. 2. Comparison of basic morphological parameters between the two cultivars, A represents cultivar Fozhi, and B represents cultivar Maling.

For the first ratio (= seed width/length), cultivar Fozhi was significantly smaller than cultivar Maling (Fig. 3a); whereas for the second ratio (= seed 
thickness/width), cultivar Fozhi was significantly larger than cultivar Maling (Fig. 3b). It implies that the $3-\mathrm{D}$ profile of cultivar Fozhi is more round than cultivar Maling. Apparently, cultivar Maling appears to be flatter in 2-D profile and is rather obovate, while cultivar Fozhi is near-elliptic..
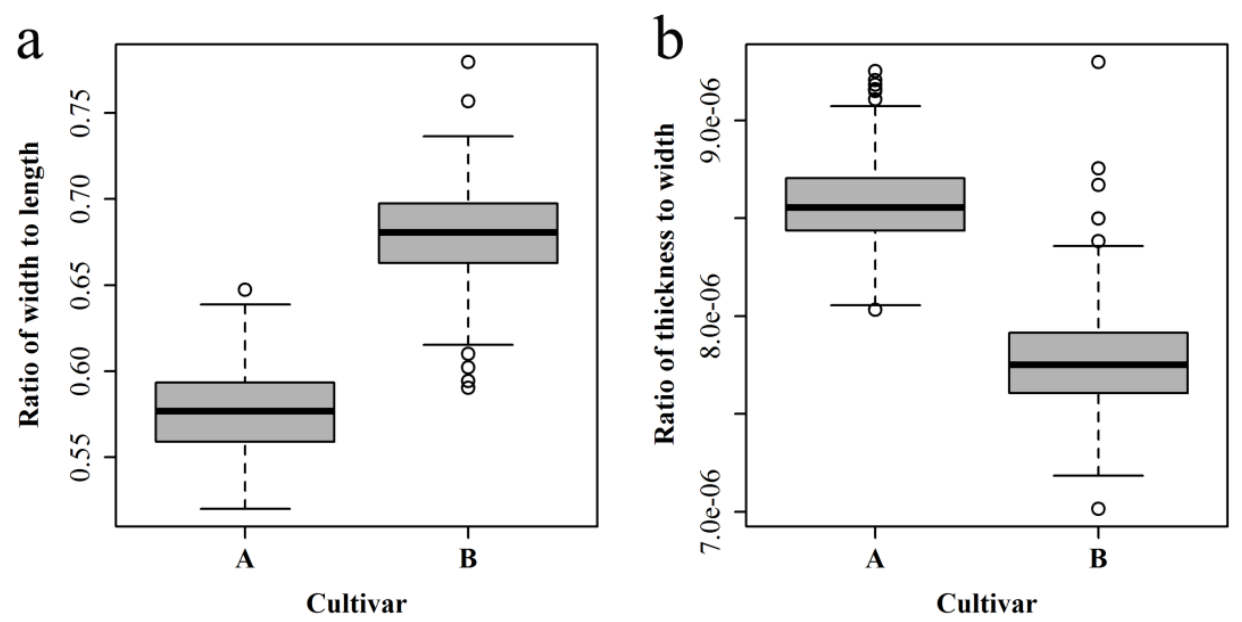

Fig. 3. Comparison between the parameters of ratios, A represents cultivar Fozhi, and $\mathrm{B}$ represents cultivar Maling.

Fig. 4a exhibits the comparison of the allometric relationship between seed fresh weight and projected area for the two cultivars. The slope estimate for Fozhi was 1.73 with the $95 \%$ CI $(1.54,1.93)$, and that of cultivar Maling was 1.91 with the $95 \%$ CI $(1.70,2.14)$. These two slope estimates were not significantly different because the $95 \%$ CI of the difference of slope bootstrap replicates includes 0 ; see Fig. 4b. 

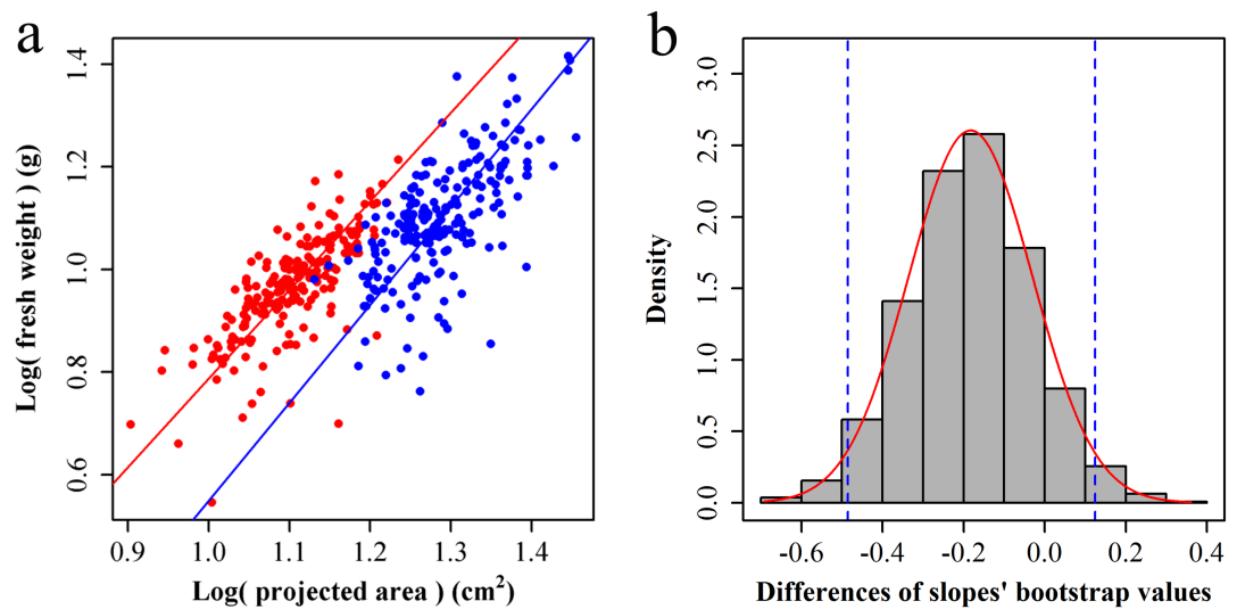

Fig. 4. Comparison of the allometric relationships between projection areas and fresh weights in the two cultivars (a) red one represents cultivar Fozhi, blue one represents cultivar Maling; (b) differences of the slopes' bootstrap values.

\section{Discussion}

In the taxonomic system of botany, G. biloba is an ancient relic plant and the only extant representative of Ginkgophyta (Šmarda et al., 2016; Zhao et al., 2016). Because of its vital economic value, more and more ginkgo cultivars are cultivated by artificial breeding and grafting in recent years. With the increasing number of Ginkgo cultivars, their accurate and efficient identification becomes increasingly difficult.

At present, these Ginkgo germplasm resources are mainly classified with two methods: (i) morphological comparison (e.g. leaf shapes and seed nucleus characters) (Chen et al., 2007), and (ii) the techniques of molecular markers (Li et al., 2013; Wang et al., 2017). Though not much attention has been paid to the traditional morphological classification, it is still very useful for its convenience 
and visualization.

In this study, some significant morphology differences are found between the seeds of two ginkgo cultivars. The comparison results of the ratios (of width to length and of thickness to width of seeds) between two cultivars are different from those reported by Chen et al. (2004), whereas the comparison result in seed weight between two cultivars is in accordance with their findings...

The regularity of projection shape can represent the whole surfac morphological features of the seed to a certain extent. We used the Gielis equation to demonstrate the significant differences between the two cultivars. The $\mathrm{CV}$ values indicate that the projection shape of Fozhi is more regular than the cultivar Maling. This morphological difference is probably caused by the ovule position on the peduncle.

Zhou (1994) listed multiple growth patterns of ginkgo ovules and speculated a changing tendency that seed size increases with the reduction of fertile ovules. In addition to the reduction of the number of ovules, he also found that the pedicel disappears in the evolutionary process of Ginkgoales, a process called peramorphosis. The latest fossil evidence shows that there is a cluster of developed ovules on peduncle of the new species, G. neimengensis (sp. nov, from the Early Cretaceous Huolinhe Formation of the Huolinhe Basin, Inner Mongolia Autonomous Region, China, Xu et al., 2017).

However, unlike ancient fossils, modern ginkgo trees usually have two ovule primordia; in each peduncle a primordium dichotomizes, and only one of them 
can normally fertilize and develop into a seed (Douglas et al., 2007). Most cultivars including Maling follow this developmental pattern. However, He (1989) reported that there were many twin seeds developing independently from two adjacent ovules for cultivar Fozhi. The seed projection shape of cultivar Fozhi is more regular than that of cultivar Maling in this study, which indicates that the spatial competition of adjacent ovules might enhance the degree of regularity of seed shape.

In the process of plant ontogeny, morphological changes can be interpreted as the change of plant allometric trajectory in the view of allometry (Zhang et al., 2017). This allometric relationship can reflect the degree of morphological differences between the two populations to some extent. However, in the allometric relationship between ginkgo seed weights and projection areas, we did not find any significant differences between the two groups of the slopes.

According to the principle of similarity, the relationship between surface area and weight for an object with uniform density is an exponent, and the power is approximately 2/3 (Makarieva et al., 2004; Thompson, 1917). Obviously, the density of ginkgo seeds cannot be uniform, whereas the degrees of heterogeneity of seed density are approximate and statistically insignificant.

In the present study, the projection shape of ginkgo seeds is well fitted by a re-parameterized Gielis equation. This equation helped us conveniently analyze the difference in positive contours between two cultivars of seeds in two-dimensional spatial level. Furthermore, because the shape of ginkgo seeds is 
not completely regular, some important morphological parameters, such as length and width measurements, can usually be inaccurate. Such measuring errors can be undoubtedly reduced to a certain extent by fitting a Gielis equation to the theoretical shape.

This is not the first time that the Gielis equation has been used to describe an organic shape in G. biloba. Silva et al. (2016) used the Gielis equation to generate the image of the G. biloba leaf and converted it into bioinspired printed monopole antenna (PMA). Clearly, use of the Gielis equation is an accurate and effective approach for making morphological comparisons.

New research shows that the Gielis equation can well fit the shapes of plant leaves (Lin et al., 2016; Shi et al., 2015b), bamboo culms (Wei et al., 2017) and tree-rings (Shi et al., 2015a). It provides us with a convenient, effective, and safe method in morphological comparisons.

\section{Conclusions}

In summary, there were significant morphological differences in the seeds of two ginkgo cultivars, in terms of width, thickness, and external shape. However, there were no significant differences in seed length and the exponents between seed weight and projection areas of two cultivars. The re-parameterized Gielis equation is applicable for describing the shapes of ginkgo seeds; it also can help us reduce the irregular measuring errors when comparing several seed morphological parameters. Due to limited resources, we compared seeds of only two ginkgo 
cultivars and were unable to analyze other cultivars from different provenances and origins. The merits of our methodology deserve further investigation into other Ginkgo biloba cultivar seeds or seeds of other plants

Acknowledgements This study was funded by the National Natural Science Foundation of China (No. 31400348), Postgraduate Research \& Practice Innovation Program of Jiangsu Province (No. KYCX17_0836), and the PAPD of Jiangsu Province. We are deeply thankful to Prof. Hsin-i Wu (Texas A \& M University) for his valuable help for commenting on the earlier version of this manuscript.

Conflict of Interest The authors declare that they have no conflict of interest. 


\section{References}

Bookstein FL (1996) Biometrics, biomathematics and the morphometric synthesis.

B Math Biol 58: 313-365.

Cao FL (2007) China Ginkgo biloba L. Records. China Forestry Press, Beijing, China. (in Chinese)

Chacón R (2006) Modeling natural shapes with a simple nonlinear algorithm. Int J Bifurcat Chaos 16: 2365-2368.

Chen P, He F, Qian B, Wei J, Wang L (2004) Seed types and their relative characteristics in Ginkgo biloba of China. Sci Silva Sin 40(3): 66-70. (in Chinese)

Chen QX, Cheng P, He AH (2007) Classification on germplasm resources of Ginkgo biloba L. J Jiangsu For Sci Technol 34(4): 44-47. (in Chinese)

Cheng YP, Zhang ST (2008) Review of exploitation and utilization of ginkgo leaves. Mod Agric Sci Technol 23: 95-96. (in Chinese)

Douglas AW, Stevenson DW, Little DP (2007) Ovule development in Ginkgo biloba L., with emphasis on the collar and nucellus. Int J Plant Sci 168: 12071236.

Dumais J, Harrison LG (2000) Whorl morphogenesis in the dasycladalean algae: the pattern formation viewpoint. Phil Trans R Soc Lond B 355: 281-305.

Faisal TR, Abad EMK, Hristozov N, Pasini D (2010) The impact of tissue morphology, cross-section and turgor pressure on the mechanical properties of the leaf petiole in plants. J Bionic Eng 7: 11-23. 
Gielis J (2003) A general geometric transformation that unifies a wide range of natural and abstract shapes. Am J Bot 90: 333-338.

Gielis J (2017) The Geometrical Beauty of Plants. Atlantis Press, Paris, France.

He FR. (1989) Cultivation of Ginkgo biloba L. trees. Jiangsu Sci Technol Press, Nanjing, China. (in Chinese)

Hu BY, Mao LL, Wang J, Xu AH (2014) Qualitative and quantitative analysis of Ginkgo biloba exocarp extracts. J Chin Med Mater 37(8): 1400-1403. (in Chinese)

Jahromi MK, Rafiee S, Jafari A, Bousejin MRG, Mirasheh R, Mohtasebi SS (2008) Some physical properties of date fruit (cv. Dairi). Int Agrophys 22: $221-224$.

Jensen RJ (1990) Detecting shape variation in oak leaf morphology: a comparison of rotational-fit methods. Am J Bot 77: 1279-1293.

Kincaid DT, Schneider RB (1983) Quantification of leaf shape with a microcomputer and Fourier transform. Can J Bot 61(9): 2333-2342.

Kuhl FP, Giardina CR (1982) Elliptic Fourier features of a closed contour.

Computer Graphics and Image Processing 18(3): 236-258.

Leicht-Young SA, Pavlovic NB, Grundel R, Frohnapple KJ (2007)

Distinguishing native (Celastrus scandens L.) and invasive (C. orbiculatus

Thunb.) bittersweet species using morphological characteristics. J Torrey Bot Soc 134(4): 441-450.

Li GP, Zhang CQ, Cao FL (2013) An efficient approach to identify ginkgo biloba 
cultivars, by using random amplified polymorphic DNA markers with a manual cultivar, identification diagram strategy. Genet Mol Res 12(1): 175182.

Lin SY, Zhang L, Reddy GVP, Hui C, Gielis J, Ding YL, Shi PJ (2016) A geometrical model for testing bilateral symmetry of bamboo leaf with a simplified Gielis equation. Ecol \& Evol 6(19): 6798-6806.

Mahadevan S, Park YH (2008) Modulation of cholesterol metabolism by Ginkgo biloba L. nuts and their extract. Food Res Int 41(1): 89-95.

Makarieva AM, Gorshkov VG, Li BL (2004) Ontogenetic growth: models and theory. Ecol Model 176(1): 15-26.

Mclellan T (1993) The roles of heterochrony and heteroblasty in the diversification of leaf shapes in Begonia dreigei (Begoniaceae). Am J Bot 80(7): 796-804.

Meinhardt H (1998) The algorithmic beauty of sea shells. Berlin, Germany.

Mirzaee E, Rafiee S, Keyhani AR, Djom-eh ZE, Kheiralipour K (2008) Mass modeling of two varieties of apricot (prunus armenaica L.) with some physical characteristics. Plant Omics 1(1): 37-43.

Nakanishi K (2005) Terpene trilactones from Ginkgo biloba: From ancient times to the 21st century. Bioorgan Med Chem 13(17): 4987-5000.

R Development Core Team (2015) R: a language and environment for statistical computing. R Foundation for Statistical Computing, Vienna, Austria. http://www. R-project.org/ [accessed 09.03.15]. 
Rohlf FJ (1996) Morphometric spaces, shape components and the effects of linear transformations. In Marcus LF et al. (ed) Advances in morphometrics, Plenum, New York, pp 117-129.

Sandhu HS, Shi PJ, Kuang XJ, Xue FS, Ge F (2011) Applications of the bootstrap to insect physiology. Fla Entomol 94(4): 1036-1041.

Shi PJ, Huang JG, Hui C, Grissino-Mayer HD, Tardif JC, Zhai LH, Wang FS, Li BL (2015a) Capturing spiral radial growth of conifers using the superellipse to model tree-ring geometric shape. Front Plant Sci 6: 856.

Shi PJ, Xu Q, Sandhu HS, Gielis J, Ding YL, Li HR, Dong XB (2015b) Comparison of dwarf bamboos (Indocalamus $s p$.) leaf parameters to determine relationship between spatial density of plants and total leaf area per plant. Ecol \& Evol 5(20): 4578-4589.

Silva PF, Freire RCS, Serres AJR, Silva PHDF, Silva JC (2016) Wearable textile bioinspired antenna for 2G, 3G, and 4G systems. Microw Opt Techn Let 58(12): 2818-2823.

Šmarda P, Veselý P, Šmerda J, Bureš P, Knápek O, Chytrá M (2016) Polyploidy in a 'living fossil' Ginkgo biloba. New Phytol 212(1): 11-14.

Thompson DW (1917) On growth and form. Cambridge University Press, London, UK.

Tredici PD (1991) Ginkgos and people-A thousand years of interaction. Arnoldia 51(2): $3-15$.

Van Oystaeyen F, Gielis J, Ceulemans R (1996) Mathematical aspects of plant 
modeling. Scripta Botanica Belgica 13: 7-273.

Wang X, Zhao Q, Tao Y, Xu M, Yu W, Xu L (2017) The fingerprints construction and genetic diversity analysis of 48 fruit used ginkgo cultivars based on SSR markers. Mol Plant Breed 15: 1963-1970.

Wei Q, Jiao C, Guo L, Ding YL, Cao JJ, Feng JY, Dong XB, Mao LY, Sun HH, Fen Y, Yang GY, Shi PJ, Ren GD, Fei ZJ (2017) Exploring key cellular processes and candidate genes regulating the primary thickening growth of moso underground shoots. New Phytol 214:81-96.

Xu XH, Yang LY, Sun BN, Wang YD, Chen P (2017) A new early cretaceous ginkgo, ovulate organ with associated leaves from Inner Mongolia, China and its evolutionary significance. Rev Palaeobot Palyno 244: 163-181.

Zhang BB, Diao TT, Dai MZ, Lyu GY, Chen SH (2016) Beauty pharmacology of activating blood circulation herbs and its application in modern cosmetics as plant extracts. Chin J Mod Appl Pharm 33(9): 1221-1226. (in Chinese)

Zhang J, Zhao YL, Jin H, Zhang JY, Wang YZ (2017) Morphological variability and allometric relationships of the herb Panax notoginseng in Yunnan, China. Acta Ecol Sin 37(2): 65-69.

Zhao YP, Yan XL, Muir G, Dai QY, Koch MA, Fu CX (2016) Incongruent range dynamics between co-occurring Asian temperate tree species facilitated by life history traits. Ecol \& Evol 6: 2346-2358.

Zhou ZY (1994) Heterochronic origin of Ginkgo biloba-type ovule organs. Acta Palaeontol Sin 33(2): 131-139. (in Chinese) 
Zhou ZY (2003) Mesozoic ginkgoaleans: Phylogeny, classification and evolutionary trends. Acta Bot Yunnan 25(4): 377-396. (in Chinese) 